\title{
Reactive melt processing of poly(ethylene terephthalate) waste into calcium terephthalate
}

\author{
Y. Y. $L i^{1,2}, D . G . Y a o^{1 *}, M . Q . G e^{2}$ \\ ${ }^{1}$ School of Materials Science \& Engineering, Georgia Institute of Technology, GA 30332 Atlanta, USA \\ ${ }^{2}$ College of Textile Science \& Engineering, Jiangnan University, Wuxi, 214122 Jiangsu, China
}

Received 5 May 2020; accepted in revised form 9 July 2020

\begin{abstract}
Recycling of waste poly(ethylene terephthalate) (PET) has attracted much attention in recent years because of the pressure from the environment. However, the cost-effective conversion of waste PET into value-added products by ecofriendly methods remains to be a challenge in the industry. In this work, a method for converting PET into calcium terephthalate by reactive melt processing was developed and investigated. Specifically, PET pellets were blended with calcium hydroxide powders using reactive melt processing, and the resulting mixture was converted into calcium terephthalate by simple hydrolysis in water. Physicochemical characterizations for morphologies, chemical compositions, and thermal properties were conducted to investigate intermediate components produced during the process, as well as the mechanisms of this new process. The results indicate that the effective transformation of molten PET into calcium terephthalate may be attributed to several factors, including the strong mechanical interaction during melt mixing, the thermal decomposition of PET chains to form carboxylic end groups, and the catalytic nature of calcium hydroxide in PET hydrolysis. This research can lead to a cost-effective route for the upcycling of waste PET into a value-added product that may be useful in demanding and emerging applications.
\end{abstract}

Keywords: recycling, polyethylene terephthalate, reactive processing, hydrolysis

\section{Introduction}

Poly(ethylene terephthalate) (PET) has been industrially produced at large volumes, particularly for packaging (including beverage bottles) and textile applications, because of its superior mechanical strength, thermal stability, and chemical resistance [1]. However, the enormous consumption of nondegradable PET contributes to the accumulation of solid waste at an alarming rate. Although landfill and incineration are currently used to contain or reduce the amount of waste PET, the occupation of land resources and the emission of toxic gases make them ecologically and economically unacceptable [2]. In order to promote the utilization of PET wastes, mechanical and chemical recycling have been proposed and investigated in recent years. In particular, mechanical recycling can treat waste PET on a large scale through washing, separating, and melt processing (by molding, extrusion, or fiber spinning) [3]. The reproduced PET, however, typically undergoes a significant reduction in molar mass, intrinsic viscosity, and mechanical properties, making it restricted to down-cycling production [4]. In contrast, waste PET can also be depolymerized into its monomers and then reused in synthesis. The conventional methods for depolymerization of PET are hydrolysis, glycolysis, and methanolysis. Among these methods, hydrolysis is considered to be relatively safe and cost-effective, which can be conducted in water under alkaline, acidic and neutral conditions to convert waste PET into terephthalate acid (TPA) [5, 6]. However, PET hydrolysis generally entails pure PET feedstock, and 
the isolation of TPA prolongs the operation time, even contributing to secondary pollution. Up to now, industrial utilization of hydrolysis in short reaction time, at lower energy consumption and from highly contaminated PET remains to be a challenge.

It is worth noting that terephthalate salts such as sodium, potassium, aluminum, magnesium and calcium terephthalates have attracted increasing attention in recent years. Particularly, the capability of terephthalate salts for large-scale energy storage has been demonstrated in batteries [7], and the layered structure with metal-organic frameworks endows terephthalate salts with superior performance in sensing, catalysis, biotechnology, separation and gas sorption [8]. Among the terephthalate salts reported so far, calcium terephthalate (CaTP) deserves special attention for its nontoxicity and relatively low density [9]. Previous research has also demonstrated good thermal stability and superior compatibility of CaTP with polymer molecules, allowing CaTP to be used as an effective filler for enhancing rheological and mechanical properties of polymers [10]. Nevertheless, most terephthalate salts are derived from the reaction of TPA with metal oxides, so is CaTP [11]. This leads to the complexity and high cost in preparation of CaTP because TPA has a high melting temperature $\left(\sim 300{ }^{\circ} \mathrm{C}\right)$ and low solubility in common solvents [12]. Therefore, the volume production of CaTP with a simple method at low cost continues to be highly desirable.

In this context, it can be concluded that TPA plays a crucial role in both PET hydrolysis and CaTP synthesis, but it is the major obstacle in purification and processing. On the other hand, Grause et al. [13] and Yoshioka et al. [14] have demonstrated the existence of CaTP in the pyrolysis products of PET catalyzed by $\mathrm{Ca}(\mathrm{OH})_{2}$ or $\mathrm{CaO}$ at $450^{\circ} \mathrm{C}$ in water steam. From their work, it may be inferred that a properly designed hydrolysis process of PET may serve as a useful method for the synthesis of CaTP, which can drastically simplify the operation steps. If this approach were combined with waste PET, the value would be even more far-reaching. However, the main obstacles to overcome in Grause et al.'s and Yoshioka et al.'s process are process complexity and high energy consumption with an unstable and high-temperature water steam, and yet a highly impure product. Aharoni [15], on the other hand, proposed that metal hydroxides should be capable of releasing water within the processing of molten PET, and severe hydrolysis should occur when PET is catalyzed by alkali metal hydroxides with no extra water. Therefore, it would theoretically be possible to simplify the hydrolysis of PET by mixing molten PET with dry alkali. Actually, Lipik and Abadie [16] have already realized a similar idea, and their results demonstrated that PET could be degraded when mixed with dry $\mathrm{Ca}(\mathrm{OH})_{2}$ under melt conditions. However, the purpose of Lipik and Abadie's work was to obtain TPA, and the results showed low efficiency in terms of TPA yield.

Since the melting temperature of $\mathrm{Ca}(\mathrm{OH})_{2}$ is substantially higher than that of PET, one obstacle during melt blending is the heterogeneity of reactants. In fact, the low yield of TPA in Lipik and Abadie's work may be partially attributed to their sample preparation method in which a relatively nonuniform mixture was utilized. On the other hand, reactive processing under continuous heating and high-stress mixing conditions using twin screws or twin rotors have been widely used in polymer processing [17]. Moreover, recent research has revealed that the efficiency of PET hydrolysis is highly dependent on the crushing degree of materials [18]. Therefore, it becomes apparent that reactive processing, which combines high-stress mixing with chemical reaction, may emerge into an effective method for controllable PET degradation. However, up to now, little research on PET hydrolysis has been carried out by reactive melt processing. This may be attributed to the high corrosivity of TPA [19], the high pressure required in water injection, and the low yield (decided by TPA produced). Nevertheless, these drawbacks may be mitigated or even eliminated if the focus is placed on the creation of CaTP, rather than TPA. In light of this, Benzaria et al. [20] described a method for making alkaline-earth terephthalate by mixing molten PET with anhydrous alkaline-earth metal hydroxide. However, the research was disclosed in the patent literature, and the only metal hydroxide used in the disclosed examples was sodium hydroxide. Details of operations and mechanisms remain to be investigated.

Herein, we provide a cost-effective way to obtain a large quantity of CaTP from PET waste. The operation procedures include reactive processing of molten PET with dry solid $\mathrm{Ca}(\mathrm{OH})_{2}$ in a twin-rotor batch mixer, followed by further hydrolysis in water with no additives. The process highlights the effective transformation of large-scale PET waste into a 
value-added product under a mild processing condition. A variety of characterizations were conducted to analyze the intermediates and the final product in order to uncover the underlying mechanism of this upcycling process.

\section{Experimental}

\subsection{Materials}

Calcium hydroxide (SKU\# 239232-500G) was obtained from Sigma-Aldrich, USA. Fiber/extrusion grade PET pellets of IV 0.83 were obtained from the former Kosa Company (now part of the Invista Company, Wichita, KS, USA) with a diameter of approximately $2.5 \mathrm{~mm}$ and a length of $1.5 \mathrm{~mm}$.

\subsection{Processing}

Reactive processing was performed by blending PET pellets with different amounts of $\mathrm{Ca}(\mathrm{OH})_{2}$ to obtain $\mathrm{Ca}(\mathrm{OH})_{2} / \mathrm{PET}$ composites with varied $\mathrm{Ca}(\mathrm{OH})_{2}$ weight percentage. The theoretical molar ratio of $\mathrm{Ca}(\mathrm{OH})_{2} / \mathrm{PET}$ for the production of calcium terephthalate is $1: 1$, corresponding to $28 \% \mathrm{Ca}(\mathrm{OH})_{2}$ in the mixture [14]. Accordingly, this weight percentage of $\mathrm{Ca}(\mathrm{OH})_{2}$ was chosen as one of the dosages in this study. It is further conjectured that lower weight fraction of $\mathrm{Ca}(\mathrm{OH})_{2}$ may facilitate purification of the reaction product since $\mathrm{Ca}(\mathrm{OH})_{2}$ should be consumed completely. For this reason, two lower $\mathrm{Ca}(\mathrm{OH})_{2}$ percentages were also chosen in the study: 25 and $10 \%$. The obtained composites from reactive mixing of these three mixtures are named as $28 \mathrm{Ca}-\mathrm{P}, 25 \mathrm{Ca}-\mathrm{P}$, and $10 \mathrm{Ca}-\mathrm{P}$, respectively, in the following text of this paper. The reaction was carried out at $270{ }^{\circ} \mathrm{C}$ in a batch mixer (C.W. Brabender Prep-Center fitted with twin roller blades) followed by immersing the mixture into water at room temperature, as shown in Figure 1. During blending, the chamber of the batch mixer was preheated to $270^{\circ} \mathrm{C}$, at which $\mathrm{Ca}(\mathrm{OH})_{2}$

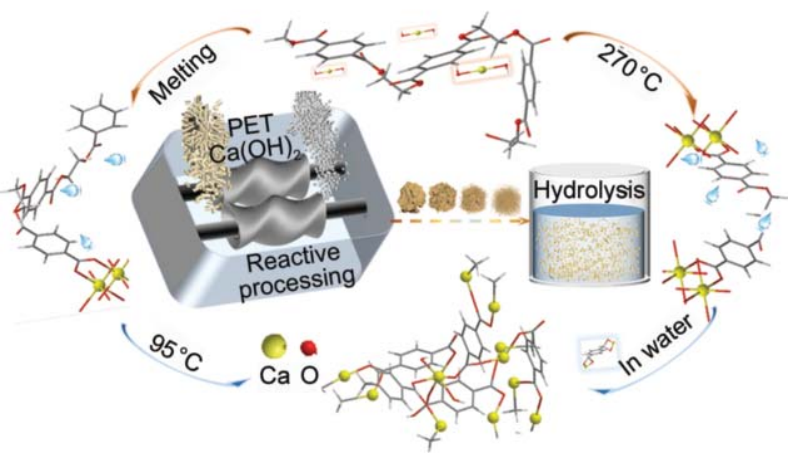

Figure 1. Schematic diagram of PET degradation and recycling. powders were added quickly into the chamber while rotation of the rotor was maintained. After the chamber temperature was equilibrated again, PET pellets were gradually added at a rotor speed of approximately $5 \mathrm{rpm}$. It took about 5 minutes to finish loading all PET pellets inside the chamber and allow PET to melt. The total mixture was left inside the mixing chamber, with the rotors running at a speed of $10 \mathrm{rpm}$ for an additional 25 minutes to ensure the completion of the reaction.

The primary experimental work was conducted on the 25Ca-P mixture. To monitor the compositional change during reactive mixing, intermediates were extracted from the mixing chamber using a small laboratory spatula. The selected intervals were 2,6 , 15 , and $25 \mathrm{~min}$, and obtained samples are referred to as $\mathrm{D}_{2 \min }, \mathrm{D}_{6 \text { min }}, \mathrm{D}_{15 \text { min }}$, and $\mathrm{D}_{25 \text { min }}$ in the following text. The final product from reactive mixing (i.e., $\mathrm{D}_{25 \text { min }}$ ) was collected, ground, and immersed in water at $95^{\circ} \mathrm{C}$ for $2-6$ hours in a convective oven. The hydrolysis product was recovered after drying the aqueous mixture at $70^{\circ} \mathrm{C}$ overnight. Intermediates during the hydrolysis process were extracted at 2, 4, and $6 \mathrm{~h}$, named as $\mathrm{H}_{2 \mathrm{~h}}, \mathrm{H}_{4} \mathrm{~h}$, and $\mathrm{H}_{6} \mathrm{~h}$. For comparison, hydrolysis was also carried out at room temperature $\left(25^{\circ} \mathrm{C}\right)$ for 15 days, and the resulting hydrolysis product is named as $\mathrm{H}_{15} \mathrm{~d}$. The $\mathrm{pH}$ value of aqueous mixtures was monitored during hydrolysis.

\subsection{Characterization}

The chemical structure of the obtained products was investigated by Fourier-transform infrared spectroscopy (FTIR) on a Nicolet ${ }^{\text {TM }}$ iS5 FTIR spectrometer (Thermo Fisher Scientific, Waltham, MA, USA) equipped with an OMNICTM software package. An Id7 ATR accessory with diamond crystal is used to scan the materials in a range of $4000-500 \mathrm{~cm}^{-1}$ by co-addition of 128 scans at a resolution of $4 \mathrm{~cm}^{-1}$. Differential scanning calorimetry instrument Q200 DSC (TA Instruments, New Castle, DE, USA) was utilized to measure the thermal properties of intermediates during the melt mixing process. Experiments were performed at a constant nitrogen flow rate of $50 \mathrm{ml} / \mathrm{min}$ by loading $10 \mathrm{mg}$ of sample material into an aluminum capsule and heating from room temperature to $300^{\circ} \mathrm{C}$ to eliminate the thermal history of the sample to be measured. After that, the sample was repeatedly heating and cooling between 50 and $300^{\circ} \mathrm{C}$ at a rate of $5^{\circ} \mathrm{C} / \mathrm{min}$ for several times, during which data were collected. For comparison 
purposes, a physical mixture of $2 \mathrm{mg} \mathrm{Ca}(\mathrm{OH})_{2}$ and $6 \mathrm{mg}$ PET ground powders (corresponding to the same mixing ratio in the $25 \mathrm{Ca}-\mathrm{P}$ sample) was included in DSC measurements, and the heating and cooling cycle was repeated for 5 times. Between each cycle, a 5 minute isothermal dwelling at $300^{\circ} \mathrm{C}$ was performed.

Thermal gravimetric analysis (TGA) was performed on a thermogravimetric analyzer (TA Instruments, New Castle, DE, USA). Samples of $6.5 \mathrm{mg}$ in mass were heated from 50 to $800^{\circ} \mathrm{C}$ with a heating rate of $10^{\circ} \mathrm{C} / \mathrm{min}$ in a nitrogen atmosphere.

The surface and appearance of intermediate products harvested during the course of the reaction were observed using an Olympus BX51 optical microscope (Olympus Corp. of the Americas, Center Valley, PA, USA) installed with an Olympus UC30 digital camera.
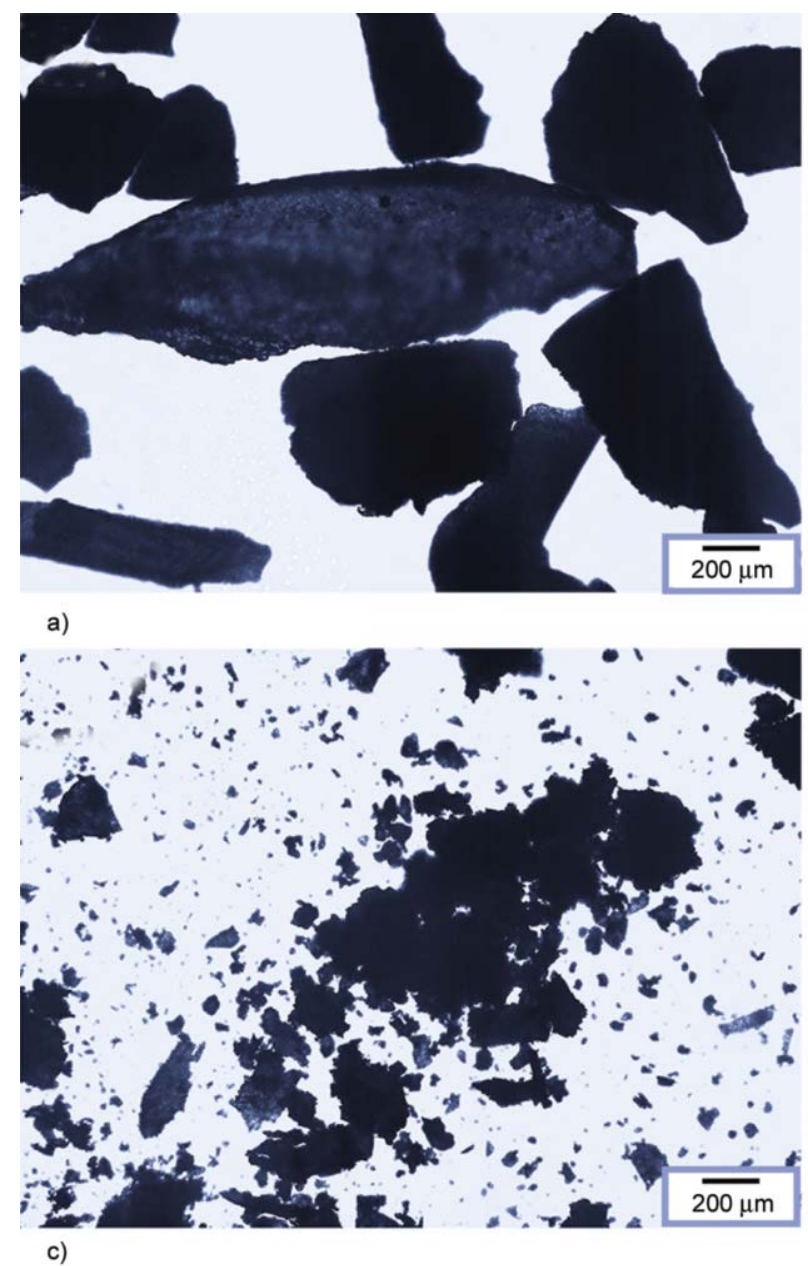

\section{Results and discussion}

\subsection{Reactive processing}

\subsubsection{Phenomenon and morphology}

Optical micrographs of intermediates produced during the reactive process of 25Ca-P are shown in Figure 2. At the first two minutes of reaction, PET pellets are heated to a molten state, and the molten sample of $\mathrm{D}_{2 \text { min }}$ becomes irregularly shaped solid after cooling in the air. As shown in Figure 2a, the sample harvested at $2 \mathrm{~min}$ is quite rigid and it is hard to fracture it into small pieces. The mixing torque fluctuates irregularly, because of the heterogeneous reactants at the beginning of reactive processing. When it is heated for six minutes inside the chamber, the mixture is transferred into a viscous material, with an appearance close to that of a suspension or solution. The mixing torque grows steadily in the reaction stage. A small amount of gas begins to evaporate at the same
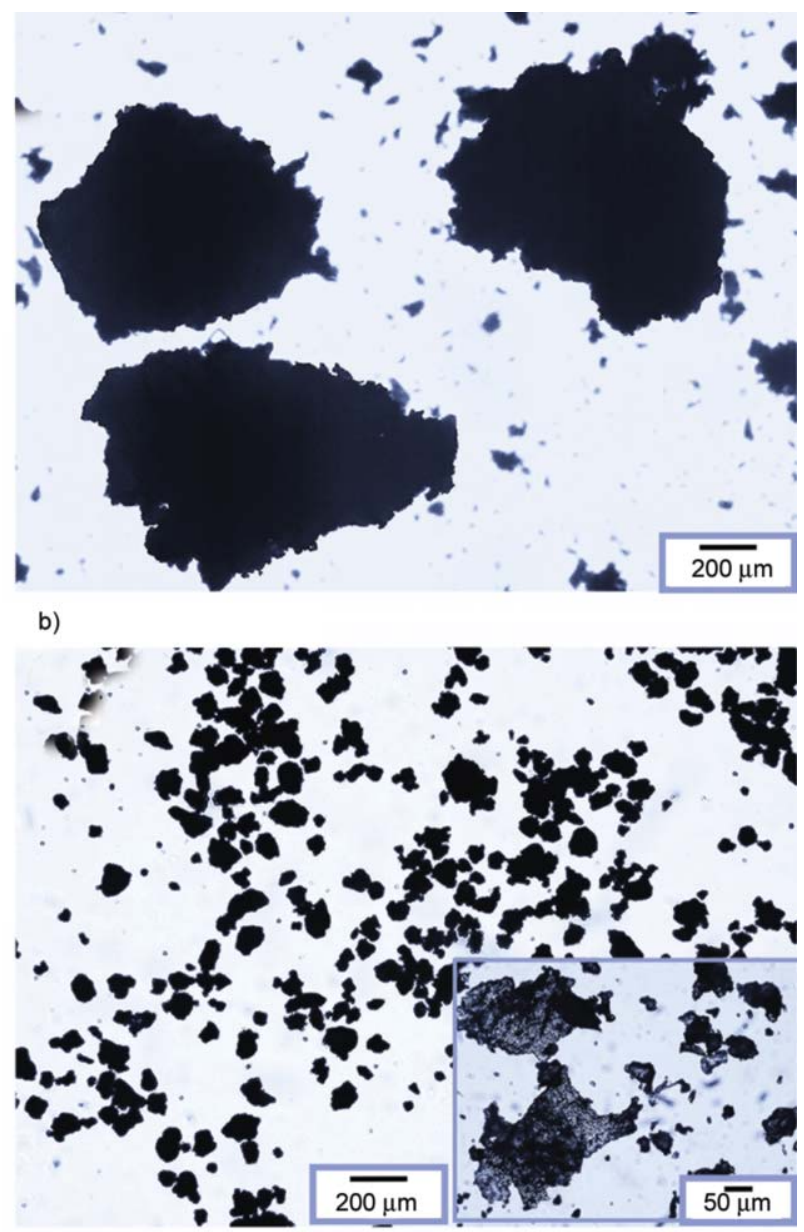

d)

Figure 2. Optical micrographs of $25 \mathrm{Ca}-\mathrm{P}$ intermediates harvested at different times during reactive processing: (a) $\mathrm{D}_{2}$ min; (b) $\mathrm{D}_{6 \text { min }}$; (c) $\mathrm{D}_{15 \text { min }}$; (d) $\mathrm{D}_{25 \text { min. }}$. 
time. This can be explained by the chemical reaction between reactants after continuous mixing at $270{ }^{\circ} \mathrm{C}$. The gas evaporation may be attributed to the evaporation of ethylene glycol, which lasts for about a couple of minutes and then diminishes. Meanwhile, the increasing torque presents a downward trend after the evaporation of gas. Observation of this sample after it is cooled to room temperature reveals a porous surface with broken edges, as shown in Figure 2b. The porosity is likely caused by the evaporation of water and gas from the inside of reactants, as well as additional hydrolysis taking place during reactive mixing. After 15 minutes of reaction, the mixing torque declines gradually as reaction proceeds, suggesting lower binding force between reactants. As shown in Figure 2c, the mixture turns into a solidlike material inside the chamber, which can be cracked with hand forces into smaller pieces after removal from the chamber. These broken pieces lose plasticity totally and become rather fragile. Meanwhile, the mixing torque has been stable at a low value. Figure $2 \mathrm{~d}$ displays the morphology of the final powders from crushing the sample harvested at 25 minutes. The average diameter of the obtained sample is measured to be $32 \mu \mathrm{m}$ under a microscope. As shown by the magnified inset in Figure 2d, these fine powders have a flake-like appearance. These observations of morphological changes indicate that effective chemical changes must have occurred in PET molecules. In addition, the transformation from PET pellets to fine powders indicates the significant effect of reactive processing.

\subsubsection{FTIR analysis}

FTIR spectra of reaction intermediates of $25 \mathrm{Ca}-\mathrm{P}$ were obtained to investigate its chemical changes during reactive processing. Original spectra of PET and $\mathrm{Ca}(\mathrm{OH})_{2}$ were measured separately for comparison. As shown in Figure 3, the spectrum of the sample at the end of the first two minutes of reaction is highly consistent with that of PET, indicating the mechanical blending of reactants with no chemical reaction. When the reaction time extends to $6 \mathrm{~min}$ utes, the asymmetric vibration of $\mathrm{COO}^{-}$exhibits a doublet at 1601 and $1576 \mathrm{~cm}^{-1}$, which become more intense with increasing reaction time. Meanwhile, the symmetric vibration of $\mathrm{COO}^{-}$at $1399 \mathrm{~cm}^{-1}$ follows the same trend, and it splits into two smaller peaks when the reaction is carried out for 15 minutes. Based on these observations, it can be concluded

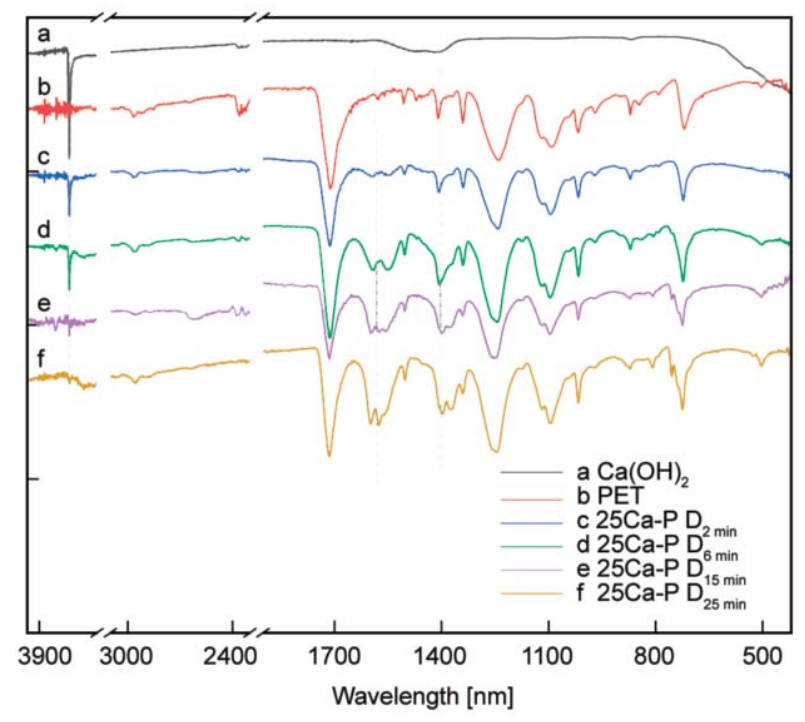

Figure 3. FTIR spectra of reactants and intermediates during reactive processing.

that part of the PET macromolecular chain is cracked, and some carboxylates are formed as the reactive process continues. Meanwhile, the absence of the broad carboxylic acid $\mathrm{O}-\mathrm{H}$ vibration at $2500 \sim 3000 \mathrm{~cm}^{-1}$ and the carboxylic $\mathrm{C}=\mathrm{O}$ vibration at $1675 \mathrm{~cm}^{-1}$ exclude the existence of terephthalic acid $[10,19]$. Therefore, doublet of asymmetric $\mathrm{COO}^{-}$ stretching may be correlated to the coordination of calcium with carboxylate, as reported in the literature [21]. The chemical reaction of PET and $\mathrm{Ca}(\mathrm{OH})_{2}$ can also be supported by the weakened $\mathrm{O}-\mathrm{H}$ vibration band in $\mathrm{Ca}(\mathrm{OH})_{2}$ observed at $3642 \mathrm{~cm}^{-1}$. The adsorption is almost absent from the spectrum of samples $D_{15}$ min and $D_{25}$ min. However, other bands in PET structure remain basically unchanged during the melt mixing process, such as the stretching vibration of $\mathrm{C}=\mathrm{O}$ at $1715 \mathrm{~cm}^{-1}$, the ester $\mathrm{C}-\mathrm{O}$ located at 1243 , and $1097 \mathrm{~cm}^{-1}$, the benzene ring peaked at 1407,872 and $720 \mathrm{~cm}^{-1}$ [22]. It is reasonable to assign the unchanged group to be fragments of PET, and take them into account in the following analysis.

\subsubsection{Thermal studies}

The thermal properties of intermediates in the reactive process of $25 \mathrm{Ca}-\mathrm{P}$ were measured by TG and DTG. Three main weight loss stages can be generally observed in the DTG curves of all samples in Figure 4. Concrete data of weight loss are summarized in Table 1. For the samples after 15 minutes of melt mixing, the first weight-loss stage is mostly attributed to the degradation of residual PET. Although the mass drop of $\mathrm{Ca}(\mathrm{OH})_{2}$ also happens in the same 


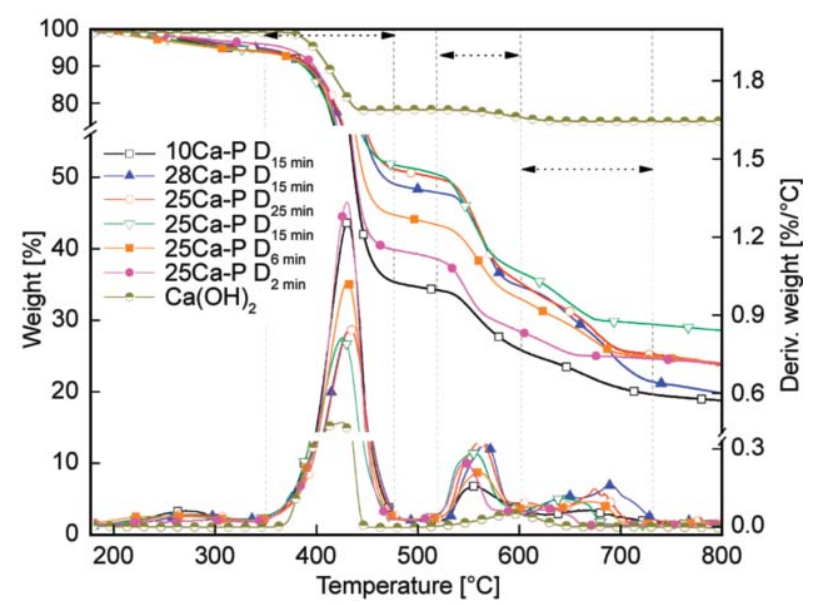

Figure 4. TG and DTG curves of reactants and intermediates during reactive processing.

temperature range, the $\mathrm{Ca}(\mathrm{OH})_{2}$ content after 15 minutes of melting has been verified to be extremely low in FTIR results. Then the second stage may be induced by the decomposition of new products in reactive processing, such as terephthalate salts or oligomers. In addition, the third stage from 629 to $753^{\circ} \mathrm{C}$ can be assigned to the pyrolysis of residual composites, viz., conversion into $\mathrm{CaCO}_{3}$ or even $\mathrm{CO}_{2}$. TGA data analysis shows that after 15 minutes of reactive mixing, the remaining percentage of PET in 10Ca-P is $58.9 \%$ and decreases to $42.2 \%$ in $25 \mathrm{Ca}-\mathrm{P}$. In comparison, the weight losses of $25 \mathrm{Ca}-\mathrm{P}$ and $28 \mathrm{Ca}-\mathrm{P}$ are roughly the same during the first two stages. These results demonstrate that the efficiency of reactive processing can be significantly enhanced by increasing $\mathrm{Ca}(\mathrm{OH})_{2}$ content. However, when the weight ratio of $\mathrm{Ca}(\mathrm{OH})_{2}$ continuously increases close to the theoretical dosage for balanced reaction $\left(28 \% \mathrm{Ca}(\mathrm{OH})_{2}\right)$, no obvious increase in conversion efficiency is achieved. Instead, when excessive $\mathrm{Ca}(\mathrm{OH})_{2}$ is present, additional difficulty in product separation may be encountered after reactive processing.

For curves of $25 \mathrm{Ca}-\mathrm{P}$ during the first six minutes of reactive processing, the impact of $\mathrm{Ca}(\mathrm{OH})_{2}$ (demonstrated by FTIR results) should be taken into consideration. Hence, the content of residual PET and new products in $\mathrm{D}_{2 \text { min }}$ and $\mathrm{D}_{6 \text { min }}$ is lower than the calculated weight loss in the first two stages. In general,

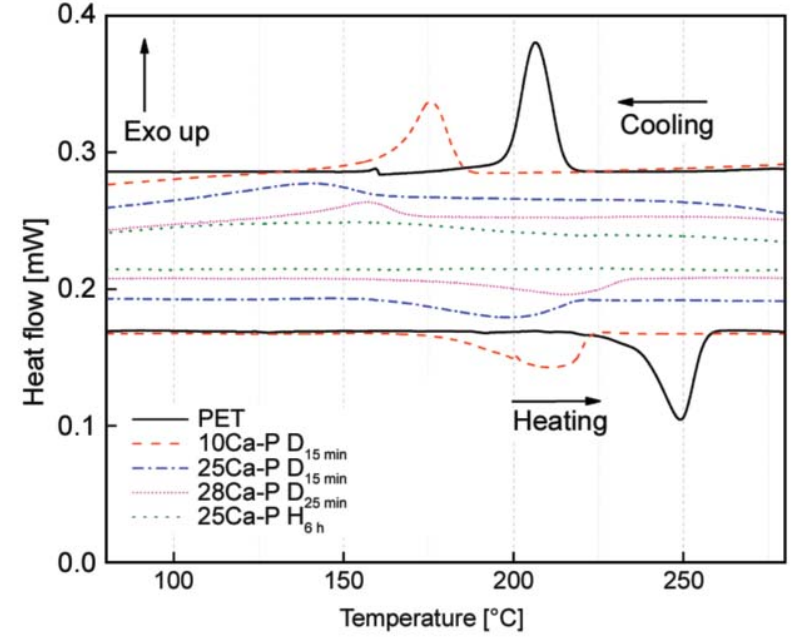

Figure 5. DSC curves of products from reactive processing and hydrolysis.

the mass drop values in the second and the third stages increase as the reaction goes on, indicating the accumulation of new products. Moreover, the TG curves of new products exhibit higher heat stability than virgin PET, suggesting the successful insertion of calcium into the long chain of PET. This finding is supported by DSC analysis as elaborated below. The DSC thermograms of Ca-P after 15 minutes of reactive processing are presented in Figure 5 in comparison with that of PET pellets. In the heating and cooling process of PET pellets, a melting point of $249^{\circ} \mathrm{C}$ and a crystallization point of $206^{\circ} \mathrm{C}$ can be observed. The two points in the curve of $10 \mathrm{Ca}-\mathrm{P}$ shift to a lower temperature. The same pattern can be found in the curve of $25 \mathrm{Ca}-\mathrm{P}$, indicating severe crack of $\mathrm{PET}$ chains in reactive processing with the addition of $\mathrm{Ca}(\mathrm{OH})_{2}$. Moreover, endothermic and exothermic peaks become broader and nearly disappear with a higher content of $\mathrm{Ca}(\mathrm{OH})_{2}$. This can be explained by the decrease of crystal regions in the residual PET after reactive processing [23].

Notably, all of the samples give no separated endothermic peak at a low temperature, suggesting no monomer or oligomer of PET existence after melting [24]. This means that the calcium in $\mathrm{Ca}(\mathrm{OH})_{2}$ may randomly replace the end groups of intermediates from PET decomposition to produce terephthalate

Table 1. Weight loss of intermediates during reactive processing in three temperature stages of TG curves.

\begin{tabular}{|c|c|c|c|c|c|c|c|}
\hline \multirow{2}{*}{$\begin{array}{c}\text { Mass loss stage } \\
{\left[{ }^{\circ} \mathrm{C}\right]}\end{array}$} & \multirow{2}{*}{$\mathrm{Ca}(\mathrm{OH})_{2}$} & \multirow{2}{*}{$10 \mathrm{Ca}-\mathrm{P} \mathrm{D}_{15 \mathrm{~min}}$} & \multirow{2}{*}{$28 \mathrm{Ca}-P \mathrm{D}_{15} \mathrm{~min}$} & \multicolumn{4}{|c|}{ 25Ca-P } \\
\hline & & & & $D_{25 \text { min }}$ & $\mathbf{D}_{15 \min }$ & $D_{6 \min }$ & $D_{2 \min }$ \\
\hline $356-484$ & $20.8 \%$ & $58.9 \%$ & $45.5 \%$ & $43.1 \%$ & $42.2 \%$ & $49.5 \%$ & $56.0 \%$ \\
\hline $522-629$ & $2.5 \%$ & $8.4 \%$ & $13.1 \%$ & $14.7 \%$ & $13.7 \%$ & $10.6 \%$ & $10.2 \%$ \\
\hline $629-753$ & $0.5 \%$ & $6.2 \%$ & $13.4 \%$ & $9.8 \%$ & $7.2 \%$ & $7.7 \%$ & $3.4 \%$ \\
\hline
\end{tabular}


salts [25]. Accordingly, the crystallization and melting temperatures of the new products from chain scission are expected to be higher than the DSC detection range of $300^{\circ} \mathrm{C}[10]$.

\subsection{Further hydrolysis in water}

\subsubsection{Microscopic characterization}

Optical micrographs of intermediates during hydrolysis of $25 \mathrm{Ca}-\mathrm{P}_{25} \mathrm{~min}$ are shown in Figure 6. When the sample is hydrolyzed in water at room temperature, the product obtained after 15 days presents no obvious change in shape, as shown in Figure 6a. Small agglomerates like 'dark spots' may be ascribed to insufficient stirring or the growth of new crystals. On the contrary, when the hydrolysis process is carried out at $95^{\circ} \mathrm{C}$ in water, morphologies of intermediates present distinct changes. After 2 hours of hydrolysis, the growth of crystallites in different directions from the boundaries of material particles can be clearly observed, as shown in Figure 6b. The phenomenon may result from the degradation of residual PET wrapped around the new products, or due to the accumulation of crystallites in the products. When the hydrolysis is prolonged to four hours, more needlelike crystals with longer lengths are observed. As shown in Figure 6c, the magnification of residual plots presents a three-dimensional growth of crystallites with a flake-like edge. It is obvious in Figure $6 \mathrm{~d}$ that the linkage between the crystallites and the flake diminishes after 6 hours of hydrolysis. Nearly all dark spots in the sample of $25 \mathrm{Ca}-\mathrm{P}_{25}$ min have been transformed into needle-like crystals after six hours of hydrolysis. These results demonstrate that the residual PET in the reaction products after reactive processing can be hydrolyzed in hot water. In addition, the increase of hydrolysis time will facilitate the growth of needle-like crystals in the hydrolysis product.

\subsubsection{FTIR analysis}

FTIR was performed to investigate the chemical changes of $25 \mathrm{Ca}-\mathrm{P} \mathrm{D}_{25}$ min during the hydrolysis
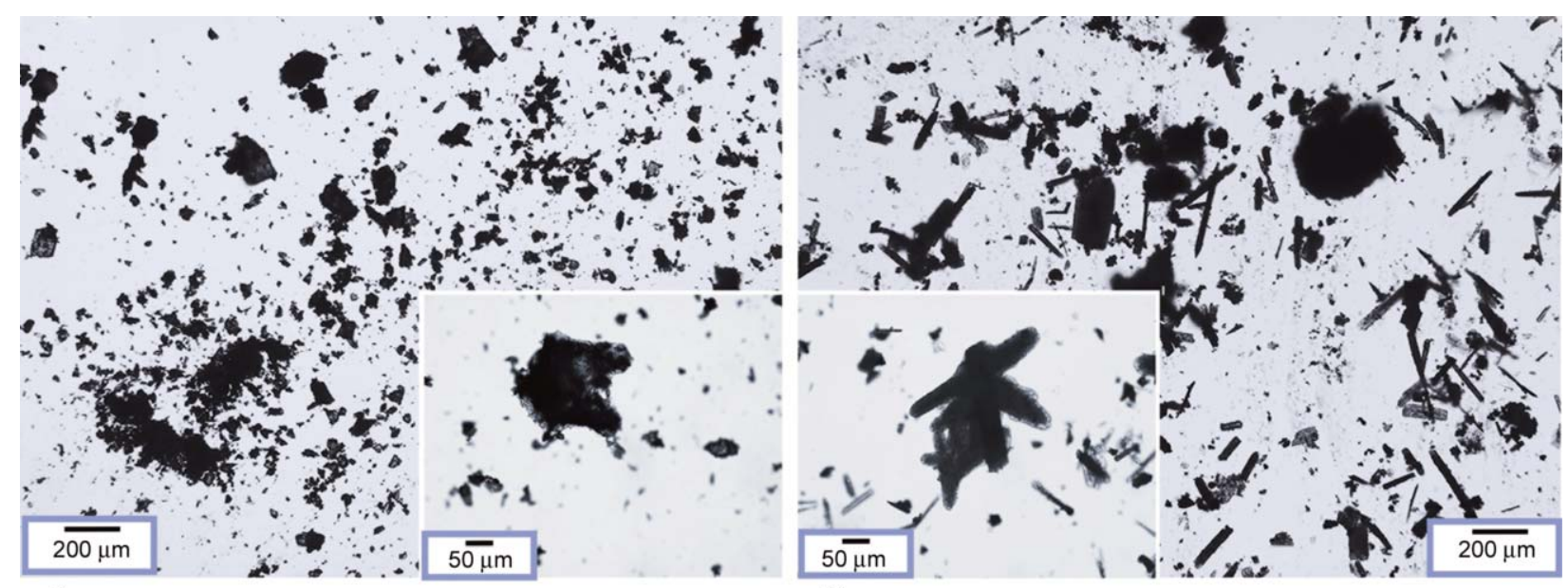

a)

b)
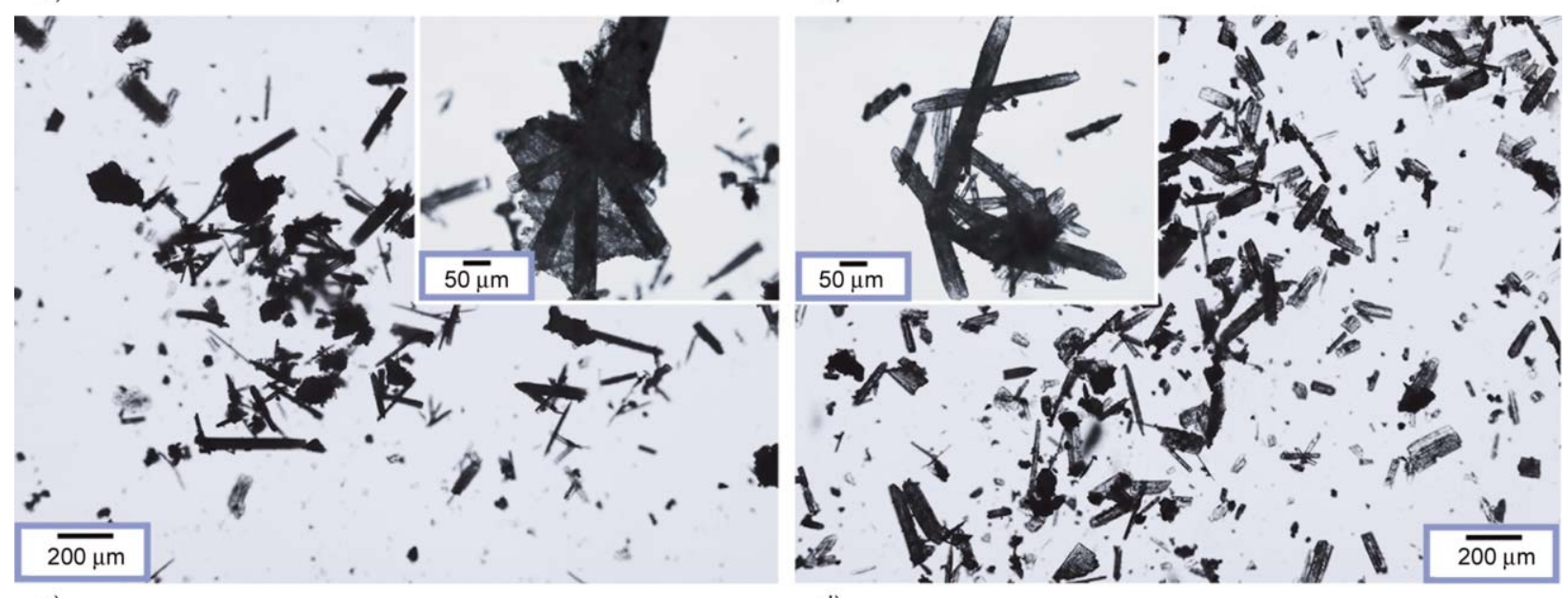

d)

Figure 6. Microscopic observation of 25Ca-P hydrolysis intermediates. (a) $\mathrm{H}_{15}$ d; (b) $\mathrm{H}_{2}$ h; (c) $\mathrm{H}_{4}$ h; (d) $\mathrm{H}_{6}$ h. 
process. Spectra of PET pellets and $25 \mathrm{Ca}-\mathrm{P} \mathrm{D}_{25}$ min prior to hydrolysis are included for comparison purposes, as shown in Figure 7. It is noticed that the vibration of $\mathrm{C}=\mathrm{O}$ in PET molecules (appearing at $1715 \mathrm{~cm}^{-1}$ ) diminishes strikingly after hydrolysis for two hours. Conversely, the asymmetric $\left(1576 \mathrm{~cm}^{-1}\right)$ and symmetric $\left(1349 \mathrm{~cm}^{-1}\right)$ stretching of $\mathrm{COO}^{-}$become stronger. These changes indicate that the carbonyl groups in PET chains have been attacked during hydrolysis [26]. Meanwhile, the vibration of $\mathrm{C}-\mathrm{O}$ shifts towards a higher wavelength and then combines with the symmetric carbonyl group of $\mathrm{COO}^{-}$ to form a broader adsorption band. The doublet at 500 and $530 \mathrm{~cm}^{-1}$ becomes more obvious after hydrolysis, which is associated with the bending vibrations of $\mathrm{Ca}-\mathrm{O}$ [10]. Therefore, the transformation can be ascribed to the interaction between calcium ions and carbonyl groups in PET to produce terephthalate salts. The aromatic in-plane and out-plane bending vibrations of $\mathrm{C}-\mathrm{H}$ appear at 1147, 1095, 1022 , and $754 \mathrm{~cm}^{-1}$, and the carboxylic deformation adsorbs at $810 \mathrm{~cm}^{-1}$ suggest the formation of CaTP in the hydrolysis process [19]. Notably, no obvious vibrations of surface water and coordinated water are observed at $3100-3500 \mathrm{~cm}^{-1}$ in the FTIR curves of hydrolysis products. According to the reports by Dominici et al. [10], and Wang et al. [7], the absence of coordinated water and the double peaks of $\mathrm{Ca}-\mathrm{O}$ exclude the presence of CaTP $\cdot 3 \mathrm{H}_{2} \mathrm{O}$. The coordination number of $\mathrm{Ca}^{2+}$ in anhydrous CaTP is 6, with all oxygen atoms originating from linkers $[9,27]$. The separation between asymmetric and symmetric stretching

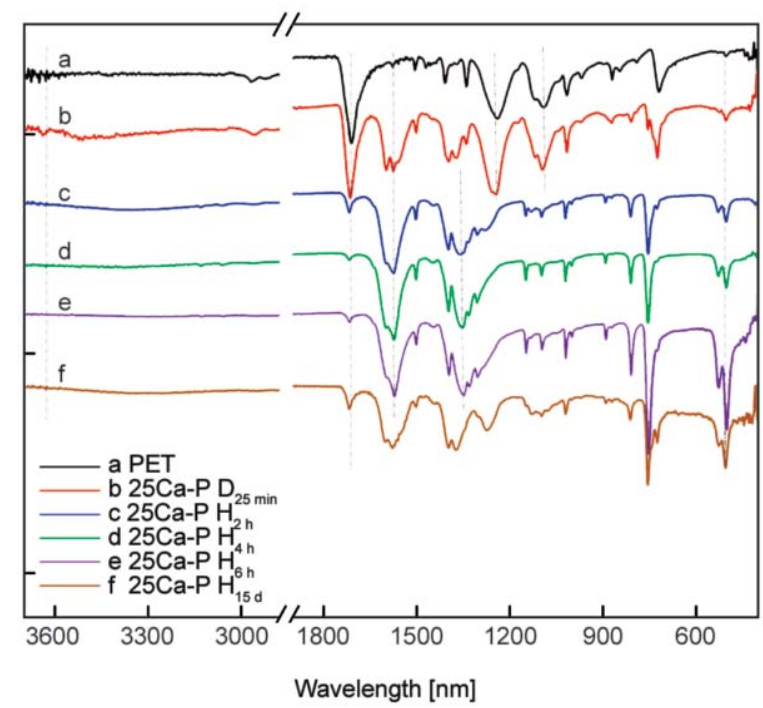

Figure 7. FTIR curves of reactants and intermediates during hydrolysis. of carboxylate is $227 \mathrm{~cm}^{-1}$, suggesting the monodentate connection between $\mathrm{Ca}^{2+}$ and $\mathrm{COO}^{-}[9,28,29]$. These combined results demonstrate that hydrolysis in hot water is an effective method for transforming the reactively processed product into CaTP.

In contrast, the band of $\mathrm{C}=\mathrm{O}\left(1715 \mathrm{~cm}^{-1}\right)$ in the sample of $\mathrm{H}_{15 \mathrm{~d}}$ remains to be obvious, suggesting the low efficiency of hydrolysis at room temperature. However, the existence of CaTP can also be observed in the sample hydrolysis at room temperature for 15 days. Although the reaction takes a long time with low efficiency, it reveals that the reactively processed $\mathrm{PET} / \mathrm{Ca}(\mathrm{OH})_{2}$ mixture can degrade in an aqueous environment under ambient conditions.

\subsubsection{Thermal studies}

TG and DTG were measured to evaluate the thermal properties of intermediates during the hydrolysis. From the DTG curves shown in Figure 8, the weight loss can be roughly divided into three stages. The corresponding weight loss values are listed in Table 2. The mass losses corresponding to the first temperature stage are close to the percentage of residual PET in intermediates without $\mathrm{Ca}(\mathrm{OH})_{2}$. Moreover, the accumulation of products can be observed by increasing weight loss in the other two temperature stages. When the sample of 25Ca-P $D_{25}$ min was hydrolyzed in hot water for two hours, the weight loss of PET decreases dramatically from 43.2 to $13.4 \%$. After that, the hydrolysis proceeds at a slower speed as the reaction time is prolonged to four to six hours. Thus, substantial hydrolysis of residual PET mainly takes place within the first two hours. After 6 hours of hydrolysis, weight loss of PET in the first temperature

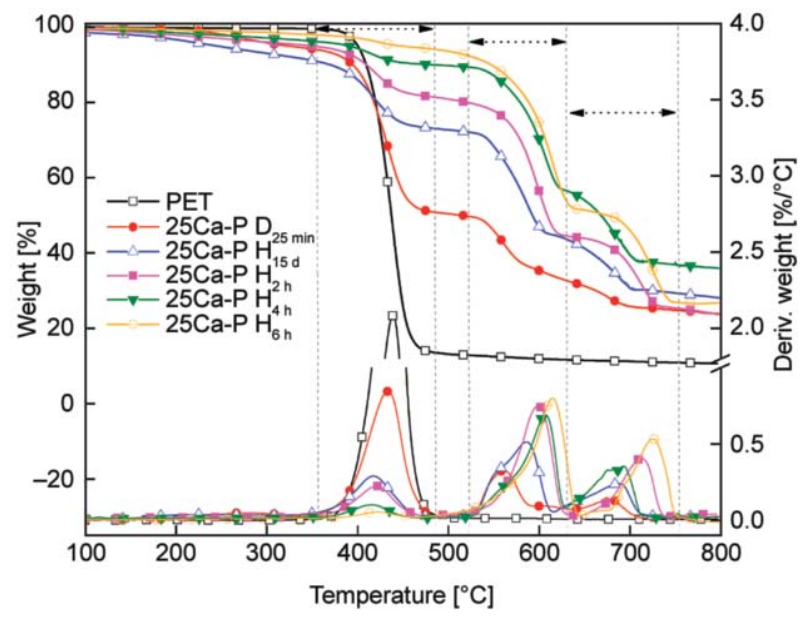

Figure 8. TG and DTG curves of reactants and intermediates during hydrolysis. 
Table 2. Weight loss of hydrolysis intermediates in three weight loss stages of TG curves.

\begin{tabular}{|c|c|c|c|c|c|c|}
\hline \multirow{2}{*}{$\begin{array}{c}\text { Temperature stage } \\
{\left[{ }^{\circ} \mathrm{C}\right]}\end{array}$} & \multirow{2}{*}{ PET } & \multirow{2}{*}{$25 \mathrm{Ca}-\mathrm{P} \mathrm{D}_{25 \mathrm{~min}}$} & \multicolumn{4}{|c|}{$25 C a-P D_{25} \min$} \\
\hline & & & $\mathrm{H}_{15 \mathrm{~d}}$ & $\mathbf{H}_{2 \mathrm{~h}}$ & $\mathbf{H}_{4 \mathrm{~h}}$ & $\mathrm{H}_{6 \mathrm{~h}}$ \\
\hline $349-476$ & $85.9 \%$ & $43.2 \%$ & $17.8 \%$ & $13.4 \%$ & $6.2 \%$ & $3.8 \%$ \\
\hline $518-602$ & $1.1 \%$ & $16.9 \%$ & $26.7 \%$ & $35.4 \%$ & $31.8 \%$ & $40.4 \%$ \\
\hline $602-733$ & $0.7 \%$ & $7.9 \%$ & $14.9 \%$ & $18.7 \%$ & $19.3 \%$ & $25.2 \%$ \\
\hline
\end{tabular}

range decreased to $3.8 \%$, indicating a low concentration of residual PET fractions in the final products. Furthermore, TG and DTG curves of the final product have high coordination with those of CaTP in the literature [10]. The mass drop of $40.4 \%$ in the second stage can be explained by the decomposition of CaTP. The next loss of $25.2 \%$ in a higher temperature range may be attributed to the decarboxylation of $\mathrm{CaCO}_{3}$ generates from the second stage $[10,30]$. Meanwhile, the melting temperature at the peak maximum of DTG curve shifts to a higher value during the hydrolysis, suggesting that the massive insertion of calcium transforms PET into a large amount of CaTP. These results are remarkably consistent with those in FTIR analysis, suggesting the main composition of the hydrolysis product to be CaTP with high purity. Notably, no obvious weight loss of $25 \mathrm{Ca}-\mathrm{P} \mathrm{D}_{25}$ min and $25 \mathrm{Ca}-\mathrm{P} \mathrm{H}_{6} \mathrm{~h}$ was observed at a temperature below $200^{\circ} \mathrm{C}$, suggesting the absence of molecular or coordinated water in the final products of reactive processing and hydrolysis.

For the sample hydrolyzed in water at room temperature, the degradation of residual PET is still ongoing. In Figure 8, the accumulation of new products is also observed in the sample after 15 days of reaction. Despite the low transformation rate, the low energy consumption of room-temperature hydrolysis may be considered as an advantage in PET recycling.

The DSC curve of 25Ca-P after hydrolysis in hot water for six hours is shown in Figure 5. The cooling and heating process presents two straight lines with no endothermic or exothermic peaks. The phenomenon can be explained by the decomposition of residual PET during the process of hydrolysis. The hydrolysis product with no melting and crystallization peaks below $300^{\circ} \mathrm{C}$ excludes the existence of PET oligomers, which is supposed to be CaTP.

\subsection{Mechanisms}

To verify the importance of mechanical melt mixing in the reactive process, a comparative experiment with physically mixed PET and $\mathrm{Ca}(\mathrm{OH})_{2}$ ground powders was performed by DSC without continuous melt mixing. The thermal curves of intermediates during the degradation of this comparative sample are shown in Figure 9. The melting point of the sample increases from 237 to $241^{\circ} \mathrm{C}$ after the first heating and remains almost unchanged in the following circles. A relatively stable structure of intermediates with a higher melting point is obtained by heating in the presence of $\mathrm{Ca}(\mathrm{OH})_{2}$. Hence, during the two cycles of heating, $\mathrm{Ca}(\mathrm{OH})_{2}$ may act as a filler material wrapped by the molten PET instead of a degradation catalyst $[10,31]$. On the contrary, a decrease of enthalpy was observed in agreement with the loss of crystallinity in PET molecules [32]. This may be attributed to the thermal decomposition of PET. However, the interaction only happens in a small range and nearly ceases after five cycles. The efficiency of PET degradation is hardly equal to that observed in reactive processing, which may be attributed to insufficient contact between reactants caused by the high melting point of $\mathrm{Ca}(\mathrm{OH})_{2}\left(580^{\circ} \mathrm{C}\right)$ [17]. Therefore, continuous reactive mixing of molten PET with $\mathrm{Ca}(\mathrm{OH})_{2}$ plays an irreplaceable role in PET degradation.

Based on the properties of intermediates and the contrast between the samples obtained from different processing conditions, a reactive mechanism is proposed,

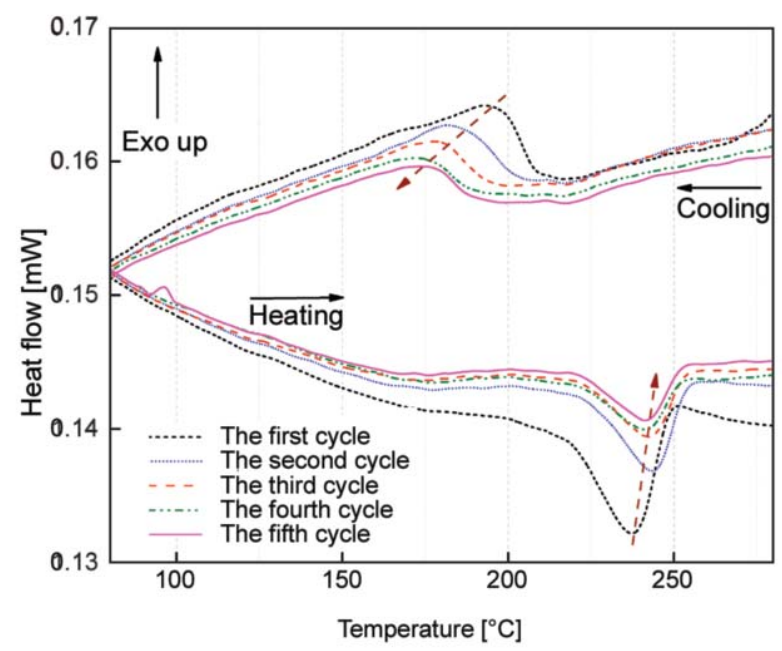

Figure 9. DSC curves of simulated degradation of $25 \mathrm{Ca}-\mathrm{P}$ without mixing. 


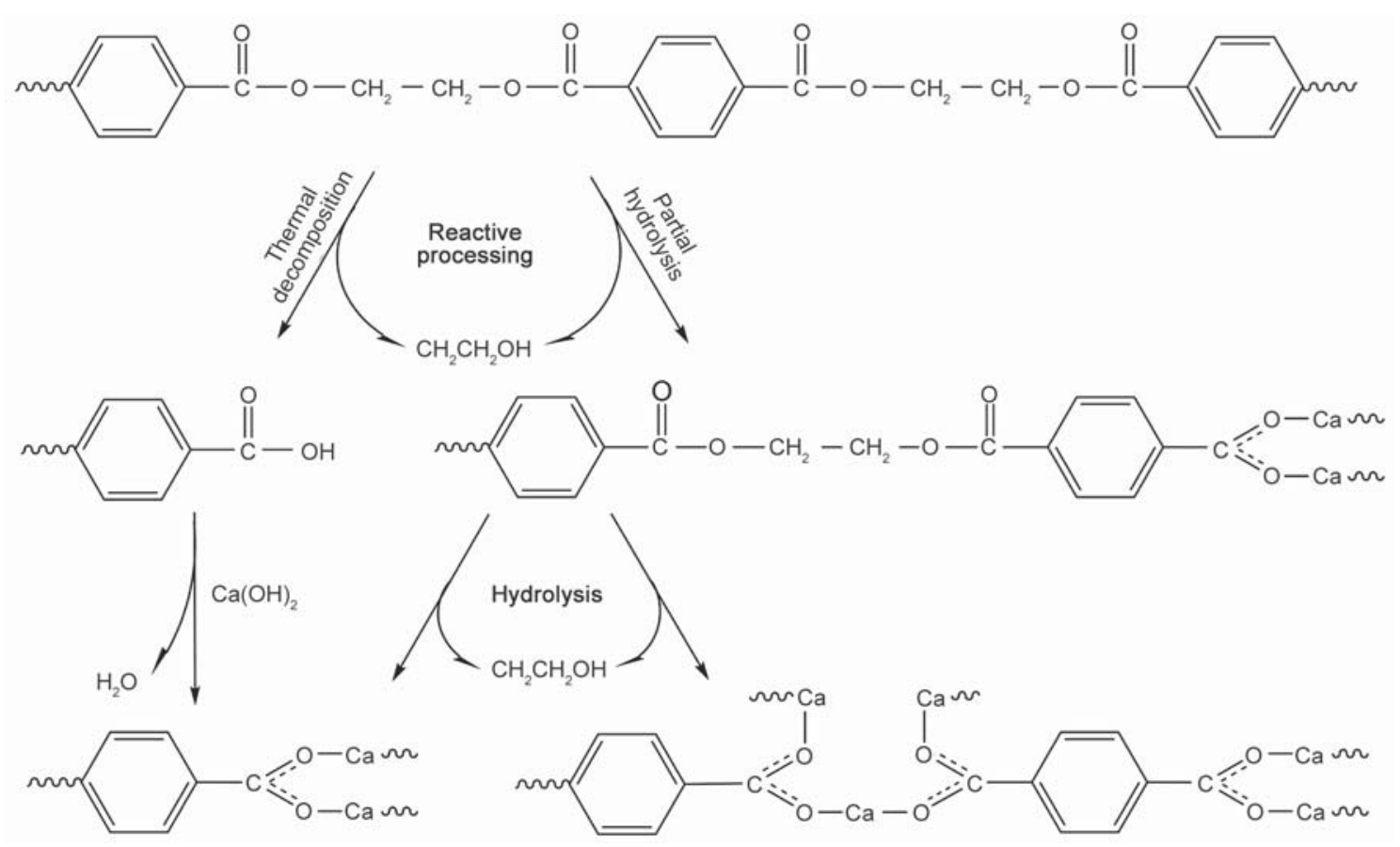

Figure 10. Proposed reaction mechanism for reactive processing and hydrolysis.

as displayed in Figure 10. During reactive processing, the content of the original PET is decreased and some heat-stable intermediates are formed. Degradation starts from the heating of the PET to a molten state [33]. Subsequently, the water released from $\mathrm{Ca}(\mathrm{OH})_{2}$ accelerates the partial hydrolysis of amorphous regions on the surface of PET. Decomposition firstly occurs at the end section of PET chains and produces terephthalate salts [16]. However, the rapid evaporation of water restricts further reactions in reactive processing. The subsequent destruction of the PET chain is maintained by thermal decomposition, resulting from the continuous heating of molten PET to form carboxyl end groups and react with $\mathrm{Ca}(\mathrm{OH})_{2}$ $[34,35]$. This is an autocatalytic process because the interaction between $\mathrm{Ca}(\mathrm{OH})_{2}$ and carboxyl groups of PET will generate water for hydrolysis. Therefore, $\mathrm{Ca}(\mathrm{OH})_{2}$ is anticipated to act both as catalysts and as reactants in the reactive process. The scission of PET long chain is actually a repeated process of calcium insertion. Intermediates with lower molecular weight and plasticity than the original PET are composed of terephthalate salts, which present to be fine powder at the end of reactive processing. However, the disordered structures of intermediates prevent them from being applied in high-value products. A cost-effective method is necessary to clean up the disordered structure of intermediate products and effectively hydrolyze the residual PET in the product of reactive processing.
The experimental results demonstrate that the continuous increase of reaction time or $\mathrm{Ca}(\mathrm{OH})_{2}$ dosages seems to be insignificant in efficiency improvement. Similarly, when the weight ratio of $\mathrm{Ca}(\mathrm{OH})_{2}$ is controlled close to but smaller than the theoretical value, no decrease in conversion efficiency is observed. The finding is consistent with the low degradation of PET after melting for 15 minutes when the reactive process comes across a bottleneck. This can be ascribed to the phase transformation of reactants (from the molten state to the solid-state) that wrap the residual PET inside of composites.

Based on the above findings, further hydrolysis of the obtained composites in water is proposed. In this way, products of reactive processing can be almost completely converted into CaTP in water. The high efficiency of hydrolysis may be attributed to a decrease in the agglomeration and increase of the reaction surface. Moreover, the water supernate of products from reactive processing has a $\mathrm{pH}$ value of 11.5 at $20^{\circ} \mathrm{C}$, providing an alkaline environment for further hydrolysis. Nevertheless, the $\mathrm{pH}$ value is lower than that of $\mathrm{Ca}(\mathrm{OH})_{2}$ saturated solution $(\mathrm{pH}=$ 12.4), indicating the residual content of $\mathrm{Ca}(\mathrm{OH})_{2}$ to be extremely low. Meanwhile, the value gradually decreases to 8 and remains constant with an increasing amount of CaTP. Hence, the alkaline environment is created by the combined properties of $\mathrm{Ca}(\mathrm{OH})_{2}$ and $\mathrm{CaTP}$. 


\subsection{Waste PET}

In this study, PET pellets were used to represent one type of waste PET with high purity for feasibility demonstration and illustration of the mechanisms. However, real PET waste from packaging (bottles), films, and fabrics contains a high content of impurities such as fillers, colorants, and additives. In this case, we think that the high selectivity of the proposed reactive processing route is useful for materials separation. Specifically, impure PET materials can be transformed into fine powders by reactive processing. Subsequently, soluble impurities can be removed from the powder mixture during hydrolysis in water to purify CaTP. Additionally, the superior thermal stability of CaTP at temperature up to $500^{\circ} \mathrm{C}$ can help promote the efficiency of purification by calcining. Nevertheless, many specific problems remain to be solved when different types of waste PET are recycled using this method. In future studies, realistic PET waste, including PET carpet waste and PET textile waste, should be incorporated in experiments to determine the effectiveness and efficiency of the method, and if necessary, additional measures will be developed for process enhancement.

\section{Conclusions}

In this study, a cost-effective method for processing waste PET into value-added CaTP is established. Molten PET was firstly blended with dry solid $\mathrm{Ca}(\mathrm{OH})_{2}$ powders through reactive processing followed by hydrolysis in water. The morphologies, chemical compositions and thermal properties of intermediates were measured and evaluated to investigate the mechanisms of the process. The results indicate that decomposition of PET during reactive processing can be attributed to the continuous melt mixing, the thermal decomposition of PET and the catalytic properties of $\mathrm{Ca}(\mathrm{OH})_{2}$. The composite material obtained from reactive mixing contains terephthalate salts with high thermal stability, which should result from the insertion of calcium in the PET chain. However, the reaction also experiences a bottleneck after 15 minutes of reactive processing due to the transformation of PET from a molten state to a solidstate. The results further show that such a reactively processed composite can be further degraded in water, especially in hot water. During this post hydrolysis, the increased contact surface of reactants and the external alkaline environment are considered to be among major factors contributing to significantly accelerated chemical conversion. The obtained product from hydrolysis was found to be CaTP, which has superior thermal stability and a needle-like crystalline morphology. Essentially, this study demonstrated a potentially cost-effective process for the conversion of waste PET into a CaTP salt that may be useful in some emerging applications.

\section{Acknowledgements}

One of the authors (Y. Y. Li) was financially supported by the China Scholarship Council (grand number 201906790046) and the Postgraduate Research \& Practice Innovation Program of Jiangsu Province (grant number KYCX20_1799).

\section{References}

[1] Badia J. D., Strömberg E., Karlsson S., Ribes-Greus A.: The role of crystalline, mobile amorphous and rigid amorphous fractions in the performance of recycled poly (ethylene terephthalate) (PET). Polymer Degradation and Stability, 97, 98-107 (2012).

https://doi.org/10.1016/j.polymdegradstab.2011.10.008

[2] Wang C-Q., Wang H., Liu Y-N.: Separation of polyethylene terephthalate from municipal waste plastics by froth flotation for recycling industry. Waste Management, 35, 42-47 (2015).

https://doi.org/10.1016/j.wasman.2014.09.025

[3] Zhou X., Wang C., Fang C., Yu R., Li Y., Lei W.: Structure and thermal properties of various alcoholysis products from waste poly(ethylene terephthalate). Waste Management, 85, 164-174 (2019).

https://doi.org/10.1016/j.wasman.2018.12.032

[4] Benvenuta-Tapia J. J., González-Coronel V. J., SorianoMoro G., Martínez-De la Luz I., Vivaldo-Lima E.: Recycling of poly(ethylene terephthalate) by chain extension during reactive extrusion using functionalized block copolymers synthesized by RAFT polymerization. Journal of Applied Polymer Science, 135, 46771/1-46771/10 (2018).

https://doi.org/10.1002/app.46771

[5] Geyer B., Lorenz G., Kandelbauer A.: Recycling of poly(ethylene terephthalate) - A review focusing on chemical methods. Express Polymer Letters, 10, 559586 (2016).

https://doi.org/10.3144/expresspolymlett.2016.53

[6] López-Fonseca R., González-Velasco J. R., GutiérrezOrtiz J. I.: A shrinking core model for the alkaline hydrolysis of PET assisted by tributylhexadecylphosphonium bromide. Chemical Engineering Journal, 146, 287-294 (2009).

https://doi.org/10.1016/j.cej.2008.09.039 
[7] Wang L., Mou C., Sun Y., Liu W., Deng Q., Li J.: Structure-property of metal organic frameworks calcium terephthalates anodes for lithium-ion batteries. Electrochimica Acta, 173, 235-241 (2015).

https://doi.org/10.1016/j.electacta.2015.05.067

[8] Wang S., McGuirk C. M., d'Aquino A., Mason J. A., Mirkin C. A.: Metal-organic framework nanoparticles. Advanced Materials, 30, 1800202/1-1800202/14 (2018). https://doi.org/10.1002/adma.201800202

[9] Mazaj M., Mali G., Rangus M., Žunkovič E., Kaučič V., Zabukovec Logar N. Z.: Spectroscopic studies of structural dynamics induced by heating and hydration: A case of calcium-terephthalate metal-organic framework. The Journal of Physical Chemistry C, 117, 7552-7564 (2013). https://doi.org/10.1021/jp311529e

[10] Dominici F., Puglia D., Luzi F., Sarasini F., Rallini M., Torre L.: A novel class of cost effective and high performance composites based on terephthalate salts reinforced polyether ether ketone. Polymers, 11, 2097/12097/14 (2019). https://doi.org/10.3390/polym11122097

[11] Mazaj M., Zabukovec Logar N.: Phase formation study of Ca-terephthalate MOF-type materials. Crystal Growth and Design, 15, 617-624 (2015).

https://doi.org/10.1021/cg501273b

[12] Singh S., Sharma S., Umar A., Mehta S. K., Bhatti M. S., Kansal S. K.: Recycling of waste poly(ethylene terephthalate) bottles by alkaline hydrolysis and recovery of pure nanospindle-shaped terephthalic acid. Journal of Nanoscience and Nanotechnology, 18, 5804 5809 (2018).

https://doi.org/10.1166/jnn.2018.15363

[13] Grause G., Sugawara K., Mizoguchi T., Yoshioka T.: Pyrolytic hydrolysis of polycarbonate in the presence of earth-alkali oxides and hydroxides. Polymer Degradation and Stability, 94, 1119-1124 (2009). https://doi.org/10.1016/j.polymdegradstab.2009.03.014

[14] Toshiaki Y., Eisaku K., Tadaaki M., Akitsugu O.: High selective conversion of poly(ethylene terephthalate) into oil using $\mathrm{Ca}(\mathrm{OH})_{2}$. Chemistry Letters, 33, 282-283 (2004). https://doi.org/10.1246/cl.2004.282

[15] Aharoni S. M.: Nucleation of PET crystallization by metal hydroxides. Journal of Applied Polymer Science, 29, 853-865 (1984). https://doi.org/10.1002/app.1984.070290314

[16] Lipik V. T., Abadie M. J. M.: Polyethylene terephthalate chemical recycling in the melted state. Polymer-Plastics Technology and Engineering, 46, 695-701 (2007). https://doi.org/10.1080/15583720701271518

[17] Yalçınyuva T., Kamal M. R., Lai-Fook R. A., Özgümüs S.: Hydrolytic depolymerization of polyethylene terephthalate by reactive extrusion. International Polymer Processing, 15, 137-146 (2000).

https://doi.org/10.3139/217.1587
[18] Raheem A. B., Noor Z. Z., Hassan A., Abd Hamid M. K., Samsudin S. A., Sabeen A. H.: Current developments in chemical recycling of post-consumer polyethylene terephthalate wastes for new materials production: A review. Journal of Cleaner Production, 225, 1052-1064 (2019).

https://doi.org/10.1016/j.jclepro.2019.04.019

[19] Kumagai S., Grause G., Kameda T., Takano T., Horiuchi H., Yoshioka T.: Decomposition of gaseous terephthalic acid in the presence of $\mathrm{CaO}$. Industrial and Engineering Chemistry, 50, 1831-1836 (2011). https://doi.org/10.1021/ie101457k

[20] Benzaria J., Dawans F., Durif-Varambon B., Gaillard J. B.: Method for recovery of alkali metal or alkaline-earth metal terephthalate and of alkylene glycol from polyethylene terephthalates. U.S. Patent 5545746, USA (1996).

[21] Achelhi K., Masse S., Laurent G., Saoiabi A., Laghzizil A., Coradin T.: Role of carboxylate chelating agents on the chemical, structural and textural properties of hydroxyapatite. Dalton Transactions, 39, 10644-10651 (2010). https://doi.org/10.1039/C0DT00251H

[22] Mendes L. C., Dias M. L., Rodrigues T. C.: Chemical recycling of PET waste with multifunctional pentaerythrytol in the melt state. Journal of Polymers and the Environment, 19, 254-262 (2011). https://doi.org/10.1007/s10924-010-0276-y

[23] de Carvalho G. M., Muniz E. C., Rubira A. F.: Hydrolysis of post-consume poly(ethylene terephthalate) with sulfuric acid and product characterization by WAXD, ${ }^{13} \mathrm{C}$ NMR and DSC. Polymer Degradation and Stability, 91, 1326-1332 (2006). https://doi.org/10.1016/j.polymdegradstab.2005.08.005

[24] Güçlü G., Yalçınyuva T., Özgümüş S., Orbay M.: Hydrolysis of waste polyethylene terephthalate and characterization of products by differential scanning calorimetry. Thermochimica Acta, 404, 193-205 (2003).

https://doi.org/10.1016/S0040-6031(03)00160-6

[25] Kumar S., Guria C.: Alkaline hydrolysis of waste poly (ethylene terephthalate): A modified shrinking core model. Journal of Macromolecular Science Part A: Pure and Applied Chemistry, 42, 237-251 (2005). https://doi.org/10.1081/MA-200050346

[26] Di Lorenzo M. L., Errico M. E., Avella M.: Thermal and morphological characterization of poly(ethylene terephthalate)/calcium carbonate nanocomposites. Journal of Materials Science, 37, 2351-2358 (2002). https://doi.org/10.1023/A:1015358425449

[27] Chen S., Lucier B. E., Chen M., Terskikh V. V., Huang Y.: Probing calcium-based metal-organic frameworks via natural abundance ${ }^{43} \mathrm{Ca}$ solid-state NMR spectroscopy. Chemistry - A European Journal, 24, 87328736 (2018).

https://doi.org/10.1002/chem.201802164 
[28]Deacon G. B., Phillips R. J.: Relationships between the carbon-oxygen stretching frequencies of carboxylato complexes and the type of carboxylate coordination. Coordination Chemistry Reviews, 33, 227-250 (1980). https://doi.org/10.1016/S0010-8545(00)80455-5

[29] Lu Y., Miller J. D.: Carboxyl stretching vibrations of spontaneously adsorbed and LB-transferred calcium carboxylates as determined by FTIR internal reflection spectroscopy. Journal of Colloid and Interface Science, 256, 41-52 (2002).

https://doi.org/10.1006/jcis.2001.8112

[30] Kumagai S., Grause G., Kameda T., Yoshioka T.: Catalytic degradation of poly(ethylene terephthalate) for benzene-rich oil recovery using metal hydroxides. Chemistry Letters, 43, 637-639 (2014).

https://doi.org/10.1246/cl.131109

[31] Gao W., Ma X., Liu Y., Wang Z., Zhu Y.: Effect of calcium carbonate on PET physical properties and thermal stability. Powder Technology, 244, 45-51 (2013).

https://doi.org/10.1016/j.powtec.2013.04.008
[32] Kao C-Y., Cheng W-H., Wan B-Z.: Investigation of alkaline hydrolysis of polyethylene terephthalate by differential scanning calorimetry and thermogravimetric analysis. Journal of Applied Polymer Science, 70, 1939-1945 (1998).

https://doi.org/10.1002/(SICI)10974628(19981205)70:10<1939::AID-APP8>3.0.CO;2-G

[33] Awaja F., Daver F., Kosior E.: Recycled poly(ethylene terephthalate) chain extension by a reactive extrusion process. Polymer Engineering and Science, 44, 15791587 (2004). https://doi.org/10.1002/pen.20155

[34] Awaja F., Pavel D.: Recycling of PET. European Polymer Journal, 41, 1453-1477 (2005). https://doi.org/10.1016/j.eurpolymj.2005.02.005

[35] Karayannidis G. P., Psalida E. A.: Chain extension of recycled poly(ethylene terephthalate) with $2,2^{\prime}-(1,4-$ phenylene)bis(2-oxazoline). Journal of Applied Polymer Science, 77, 2206-2211 (2000).

https://doi.org/10.1002/10974628(20000906)77:10<2206::AID-APP14>3.0.CO;2-D 